\title{
Mitral Valve Repair in Rheumatic Disease: Early Results
}

\author{
Pradhan S, Koiraia B, Koiraia R, Sharma J, Acharya A, Sharma $P$, \\ Rai K,
}

Mitral valve repair is an accepted method of treating severe mitral valve disease due to rheumatic fever, Valve repair confers fewer complications and better survival as compared to mitral valve replacement. Since commencing open-heart surgery at SGNHC, 49 mitral valve repair operations have been performed here. There were 25 male and 24 female patients. The average age was 28.7 years (5 - 68 years). All were symptomatic with significant dyspnoea and palpitation. Other symptoms were hemoptysis in eight, peripheral embolism in one and CNS embolism in four. Associated lesions included ASD in three, an ASD and constrictive pericarditis in one and a pseudoaneurysm of the left common iliac artery with PDA in the fifth. One patient had undergone CMV nine years before presentation. All patients other than simple OMV and pediatric age underwent peroperative trans-esophageal echocardiography to assess the quality of repair. Of the 49 mitral valve repairs, 14 underwent open mitral valvotomy and 31 had mitral valve repair. Of the 31 mitral repair patients, 29 received annuloplasty ring. Four patients were converted to mitral replacement and three underwent revision of repair during the primary surgery based on poor valve competence on per-operative TEE. Twelve patients underwent concomitant aortic valve raplacement for a co-existing significant aortic valve disease. Tricuspid valve repair was carried out in all those with severe tricuspid incompetence. One patient underwent repair revision at one month postop for early annuloplasty ring dehiscence.

Thirty-nine patients had undergone echocardiography to assess their valve function at three months. Eighteen patients $(62.06 \%)$ had trace or no mitral regurgitation; six $(20.68 \%)$ had mild regurgitation; four $(13.79 \%)$ had moderate regurgitation and two $(6.89 \%)$ had severe regurgitation. We were unable to trace the record of ten patients.

In conclusion, mitral valve repair in rheumatic mitral disease with preserved valve quality is feasible with prospects of favorable outcome.

Shahid Gangalal National Heart Center, Kathmandu, Nepal. 